\title{
ChemComm
}

Cite this: Chem. Commun., 2013, 49, 4848

Received 24th February 2013 Accepted 30th March 2013

DOI: $10.1039 / c 3 c c 41414 k$

\section{A fluorescence-switchable luminogen in the solid state: a sensitive and selective sensor for the fast "turn-on" detection of primary amine gas $\dagger$}

\author{
Tianyu Han, ${ }^{\text {ab }}$ Jacky W. Y. Lam, ${ }^{a}$ Na Zhao, ${ }^{a}$ Meng Gao, ${ }^{a}$ Zhiyong Yang, ${ }^{a}$ \\ Engui Zhao, ${ }^{a}$ Yuping Dong*b and Ben Zhong Tang*acd
}

www.rsc.org/chemcomm

The emission of pyrrole-substituted benzoic acid can be repeatedly switched between the dark and bright states in the solid state by chemical fuming and heating processes, enabling it to work as a rapid sensitive fluorescent sensor for primary amine detection.

Development of sensitive and selective sensory systems for the detection of chemical and biological agents has attracted much attention because they play important roles in industrial, environmental and biological processes and systems. ${ }^{1}$ Among them, the fluorescence (FL)-based techniques are superior to other analytical methods and enjoy advantages such as high sensitivity, low background noise and a wide working range. ${ }^{2}$ Thanks to the enthusiastic efforts of scientists and technologists, a large variety of fluorescent sensors have been developed. Many of them operate in a "turn-off" mode: an initially highly emissive dye becomes non-fluorescent when it interacts with a quenching species through a mechanism of FL resonance energy transfer. ${ }^{3}$ Few of the sensing systems function in a "turn-on" mode though they are more sensitive, less likely to generate false-positive signals and compatible with continuous monitoring of biological processes and events. ${ }^{4}$

Amines are derivatives of ammonia and are widely used in the food and dyeing industries, gas treatment plants and in medical diagnosis. ${ }^{5}$ Low molecular weight amines, however, are toxic and easily absorbed through the skin. Many high molecular weight amines, on the other hand, are biologically highly active.

\footnotetext{
${ }^{a}$ Department of Chemistry, Institute for Advanced Study, State Key Laboratory of Molecular Neuroscience, Institute of Molecular Functional Materials and Division of Biomedical Engineering, The Hong Kong University of Science \& Technology (HKUST), Clear Water Bay, Kowloon, Hong Kong, China.E-mail: tangbenz@ust.hk ${ }^{b}$ College of Materials Science and Engineering, Beijing Institute of Technology,

5 South Zhongguancun Street, Beijing, 100081, China.

E-mail: chdongyp@bit.edu.cn; Fax: +86-10-6894-8982

${ }^{c}$ Guangdong Innovative Research Team, SCUT-HKUST Joint Research Laboratory,

State Key Laboratory of Luminescent Materials and Device, South China University of Technology (SCUT), Guangzhou 510640, China

${ }^{d}$ HKUST Shenzhen Research Institute, No. 9 Yuexing 1st RD, South Area,

Hi-tech Park, Nanshan, Shenzhen, China 518057

$\dagger$ Electronic supplementary information (ESI) available: Synthesis and characterization, emission, IR and UV spectra and X-ray diffractograms. See DOI: 10.1039/ c3cc41414k
}

Thus, development of sensitive detection systems for amines has both academic value and technological application. Much work has been done in the area of developing sensors for amine detection in solution. ${ }^{6}$ For practical application, it is preferable to perform the detection on a solid support because it requires no complex and expensive spectrofluorometer and it is thus simple, quick, green and convenient. ${ }^{7}$ Unfortunately, such sensing systems are rather limited. ${ }^{8}$ In this work, we investigated the photophysical properties of a pyrrole derivative, 4-(2,5-diphenyl-1-pyrrolyl)benzoic acid (TPPA, Fig. 1A), and found that its FL can be repeatedly switched between dark and bright states by fuming with primary amine gas and heating processes, making it promising as a rapid, sensitive and selective FL chemosensor for amine detection.

TPPA was prepared according to the synthetic route shown in Scheme S1 in the ESI. $\nmid$ Detailed procedures can be found in our previous publications. ${ }^{9}$ Its structure was well characterized using IR, NMR and MS spectroscopies with satisfactory analysis results (Fig. S1 and S2, ESI $\dagger$ ). TPPA absorbs at $292 \mathrm{~nm}$ in a dilute THF solution $(10 \mu \mathrm{M})$ (Fig. 1A). When UV irradiated at $290 \mathrm{~nm}$, it emits weakly at $464 \mathrm{~nm}$. The FL quantum yield $\left(\Phi_{\mathrm{FL}}\right)$ estimated using quinine sulfate is merely $2.7 \%$, presumably due to the active rotation of its peripheral phenyl rings, which effectively consumes the energy of the excitons via nonradiative relaxation channels. The $\Phi_{\mathrm{FL}}$ value drops to $0.25 \%$ when $90 \%$ of water is added to the THF solution. Since TPPA is insoluble in

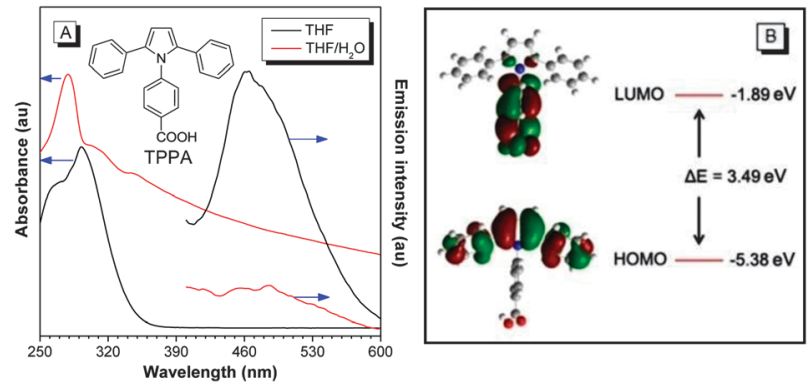

Fig. 1 (A) Absorption and emission spectra of TPPA in solution (THF; $10 \mu \mathrm{M}$ ) and aggregated states (THF-water mixture $1: 9 \mathrm{v} / \mathrm{v} ; 10 \mu \mathrm{M}$ ). Excitation wavelength: $290 \mathrm{~nm}$. (B) Molecular orbital amplitude plots of HOMO and LUMO of TPPA. 
water and a level-off tail is observed at the longer wavelength region of the UV spectrum, its molecules must have been aggregated in the aqueous mixture with such high water content. Clearly, the FL of TPPA is quenched by aggregate formation, which is exactly opposite to that observed in its sodium salt form and ester derivative. ${ }^{9}$ In the aggregated state, the rotation of the phenyl rings is restricted due to the physical constraint, which blocks the nonradiative pathways and hence enhances the FL. On the other hand, as suggested by the appearance of the carbonyl stretching vibration at $1681 \mathrm{~cm}^{-1}$ in the IR spectrum, the TPPA molecules dimerize presumably via hydrogen bonding between the carboxylic acid groups (Fig. S3A, ESI $\dagger$ ). ${ }^{10}$ When one molecule is photoexcited, an excimer-like species will be formed, whose nonradiative relaxation quenches the light emission of the luminophore. The competition between the constructive and destructive effects may consequently render TPPA non-emissive in the aggregated state.

To better understand the photophysical properties of TPPA, a theoretical calculation was carried out. The optimized molecular structure and HOMO and LUMO plots are shown in Fig. 1B. The HOMO of TPPA is dominated by the orbitals from the central pyrrole ring and the peripheral phenyl rings, while the orbitals from the benzoic acid moiety contribute mainly to the LUMO level. The energy band gap is calculated by subtracting the LUMO energy level from the HOMO energy level and is found to be equal to $3.49 \mathrm{eV}$.

The above discussion shows that dimer formation is mainly responsible for the emission quenching of TPPA in the aggregated state. If such formation is disrupted by external stimuli, its FL may be restored, making the dye molecule to serve as a "turn-on" fluorescent sensor for that analyte. Amine is capable of forming a hydrogen bond with the carboxylic acid group. With regard to this, we utilized TPPA adsorbed on a filter paper as a chemosensor for amine detection. As shown in Fig. 2A, the dye-loaded paper strip stuck on the inner wall of a quartz cell is nonemissive under $350 \mathrm{~nm}$ UV irradiation. When a few drops of primary amine liquid was added at the bottom of the cell, intense blue light was recorded, which could be readily observed by the naked eye. While the $\Phi_{\mathrm{FL}}$ value of TPPA solid powder determined by a calibrated
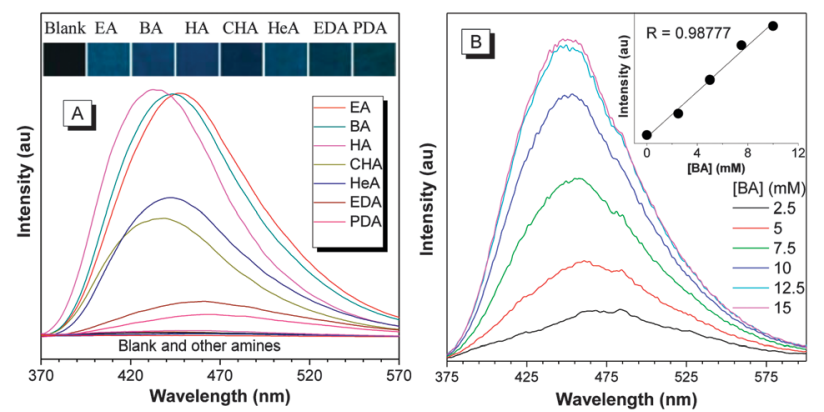

Fig. 2 (A) Fluorescent photographs and emission spectra of TPPA adsorbed on filter papers fumed by different amines. Abbreviation: ethylamine (EA), butylamine $(B A)$, hexylamine $(H A)$, cyclohexylamine $(C H A)$, heptylamine $(\mathrm{HeA})$, ethylenediamine (EDA), propanediamine (PDA), pyridine (PD), dimethylamine (DMA), diethylamine (DEA), dipropylamine (DPA), N,N-diisopropylamine (DIPA), decylamine (DA), di-n-octylamine (DOA) and triethylamine (TEA). The photos were taken under $365 \mathrm{~nm}$ UV irradiation from a hand-held UV lamp. (B) Emission spectra of TPPA drop-casting films after being dipped into aqueous solutions of butylamine with different concentrations. Inset: change in FL intensity with butylamine concentration. Excitation wavelength: $350 \mathrm{~nm}$. integrating sphere is $4.0 \%$, it jumps to $52.4 \%$ after being fumed with butylamine gas, showing a $\sim 13$-fold enhancement. On the other hand, secondary and tertiary amine gases exert no effect on the FL process of TPPA. In addition to visual observation, we also checked the FL response of the paper strip to various organic amine gases using a spectrofluorometer. Although the peak maximum varies slightly with the type of amine, the overall result is consistent with that from the visual observation and demonstrates the high selectivity of TPPA towards primary amines. Among them, those with a low molecular mass such as ethylamine and butylamine exhibit a stronger emission "turn-on" effect, probably due to their higher volatility and smaller size, which can help them to easily diffuse into the TPPA lattice without suffering severe steric hindrance.

To have a quantitative picture, we dipped TPPA drop-casting films into aqueous solutions of butylamine with different concentrations and measured their FL. As depicted in Fig. 2B, the FL spectrum was intensified upon increasing the amine concentration. The plot of emission intensity versus amine concentration gives a straight line with a detection limit of $3 \mathrm{mM}$ and a correlation coefficient of 0.9877 . The fumigation process caused no FL enhancement in the sodium salt and ester of TPPA. It also exerts no change in the mass spectrum of TPPA. These suggest that the carboxylic acid group plays an important role in the amine sensing and rule out the possibility of formation of amide from TPPA and amine vapor as its formation requires the use of a dehydrating agent and catalysts, which is unlikely, in most cases, to be triggered by such a simple chemical fuming process. The formation of a hydrogen bond between the amine molecule and the carboxylic acid group of TPPA changes the packing arrangement of the dye, as revealed by the spectral difference in its X-ray diffractograms before and after gas fumigation (Fig. S4, ESI $\dagger$ ). The absorption of the TPPA is also altered in the presence of amine gases, with the spectrum of the fumed film being red-shifted from that of the untreated one (Fig. S5, ESI $\dagger$ ). Observation using transmission electron microscopy (TEM) shows that nanofibres are formed by slow evaporation of the THF solution of TPPA at room temperature, which disintegrate into shorter and thicker cables upon exposure to butylamine gas for $40 \mathrm{~s}$ (Fig. 3). Analysis using IR spectroscopy depicts that the carbonyl absorption peak shifts to $1625 \mathrm{~cm}^{-1}$ after being fumed with butylamine gas, while no obvious peak shift was observed when treated with triethylamine gas (Fig. S3, ESI $\dagger$ ).

Stronger emission induction was observed when the TPPAembedded filter paper strips were directly dipped into the amine solutions due to the better contact of the solid papers with liquid (Fig. S6, ESI $\dagger$ ). The "turn-on" process is very fast. As shown in Fig. 4A, the relative emission intensity of the TPPA

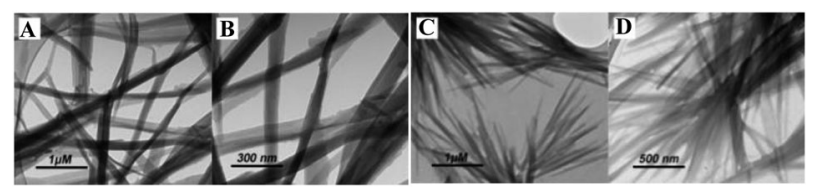

Fig. 3 TEM images of TPPA drop-casting films ( $A$ and $B$ ) before and ( $C$ and $D$ ) after being fumed with butylamine gas. 


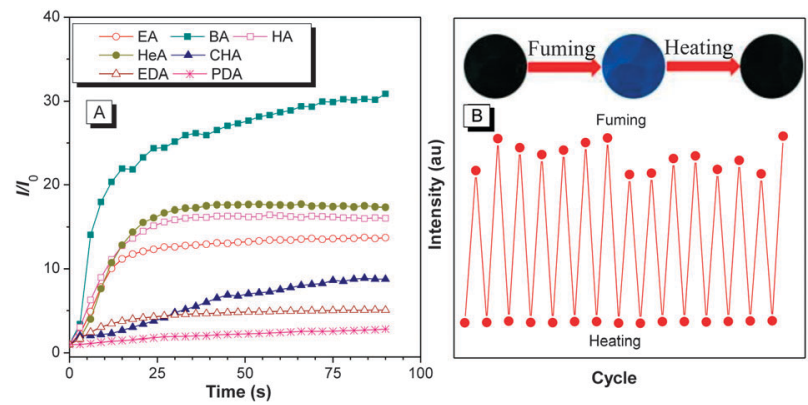

Fig. 4 (A) Change in the relative emission intensity $\left(/ / /_{0}\right)$ of TPPA films exposed to various primary amine gases with time. (B) Reversible switching of the light emission of TPPA film between dark and bright states by repeated fuming and heating processes. Excitation wavelength: $350 \mathrm{~nm}$.

film becomes progressively higher with an increase in the exposure time, and reaches its maximum value within a short time down to $30 \mathrm{~s}$. A short video was made to demonstrate the fast response of the device to butylamine gas (see ESI $\dagger$ ). In addition to being rapid, the detection method also enjoys the advantages of reversibility and reproducibility. The FL of the fumed TPPA film can be "turned-off" easily by heating the film at $100{ }^{\circ} \mathrm{C}$ for $60 \mathrm{~s}$. The "dark" and "bright" states can be interconverted to each other many times without fatigue and loss in the FL intensity, as these stimuli are nondestructive in nature (Fig. 4B).

In summary, a convenient, selective and sensitive fluorescent chemosensor has been developed. TPPA is nonemissive in both solution and solid states. It transforms into a strong fluorophore in the presence of amine, enabling it to work as a "turn-on" sensor for amine gas detection. Thus, the TPPAloaded filter paper strip becomes emissive when fumed with primary amine gases, but still remains nonemissive when exposed to other amines. The detection is rapid and strong, and can be readily observed by the naked eye due to the "lighting up" nature of the sensory process. The emission of TPPA can be switched between the dark and bright states repeatedly by fuming and heating processes, demonstrating a prototype device using TPPA for detecting primary amines in real-world applications. Since ammonia is generated during food degradation, we would like to utilize TPPA for such detection. This is currently under active investigation in our laboratory.

This work was partially supported by the National Basic Research Program of China (973 Program; 2013CB834701), National Science Foundation of China (20974028, 51073026, 51061160500 and 21074011), the RPC and SRFI Grants of HKUST (RPC11SC09 and SRFI11SC03PG), the Research Grants Council of Hong Kong (HKUST2/CRF/10 and N_HKUST620/11), the Innovation and Technology Commission (ITCPD/17-9), the University Grants Committee of Hong Kong (AoE/P-03/08), the Specialized Research Fund for the Doctoral Program of Higher Education (Grant No. 20091101110031) and the Major Project Seed Research Program of Beijing Institute of Technology (Grant No. 2012CX01008). B. Z. Tang thanks the support of the Guangdong Innovative Research Team Program of China (201101C0105067115).

\section{Notes and references}

1 (a) I. L. Medintz, M. T. Vyeda, E. R. Goldman and H. Mattoussi, Nat. Mater., 2005, 4, 435; (b) M. W. Peczuh and A. D. Hamilton, Chem. Rev., 2000, 100, 2479; (c) T. Hutai, H. Tomoda, T. Ohkawa, Y. Yabe and K. Araki, Angew. Chem., Int. Ed., 2008, 47, 9522; (d) N. A. Rakow and K. S. Suslick, Nature, 2000, 406, 710; (e) N. A. Rakow, A. Sen, M. C. Janzen, J. B. Ponder and K. S. Suslick, Angew. Chem., Int. Ed., $2005,44,4528$.

2 (a) A. P. Desilva, H. Q. N. Gunaratne, T. Gunnlangsson, A. J. M. Huxley, C. P. Mccoy, J. T. Rademacher and T. E. Rice, Chem. Rev., 1997, 97, 1515; (b) W. P. Ambrose, P. M. Goodwin, J. H. Jett, A. Van Orden, J. H. Werner and R. A. Keller, Chem. Rev., 1999, 99, 2929; (c) W. F. Patton, BioTechniques, 2000, 28, 944.

3 (a) T. Terail and T. Nagano, Curr. Opin. Chem. Biol., 2008, 12, 515; (b) S. W. Thomas, G. D. Joly and T. M. Swager, Chem. Rev., 2007, 107, 1339; (c) T. L. Andrew and T. H. Swager, J. Am. Chem. Soc., 2007, 129, 7254.

4 (a) Y. Hong, J. W. Y. Lam and B. Z. Tang, Chem. Soc. Rev., 2011, 40, 5361; (b) Y. Hong, J. W. Y. Lam and B. Z. Tang, Chem. Commun., 2009, 4332; (c) J. C. Sanchez and W. C. Trogler, J. Mater. Chem., 2008, 18, 3143; (d) E. L. Que, D. W. Domaille and C. J. Chang, Chem. Rev., 2008, 108, 1517; (e) M. Sameiro and T. Goncalves, Chem. Rev., 2009, 109, 190; $(f)$ X. Lou, D. Ou, Q. Li and Z. Li, Chem. Commun., 2012, 48, 8462 .

5 (a) J. M. Landete, B. Rivas, A. Marcobal and R. Munoz, Int. J. Food Microbiol., 2007, 117, 258; (b) V. P. Aneja, P. A. Roelle, G. C. Murray, J. Scutherland, J. W. Erisman, D. Faler, W. A. H. Asman and N. Patni, Atmos. Environ., 2001, 35, 1903; (c) P. Boeker, G. Horner and S. Rosler, Sens. Actuators, B, 2000, 70, 37; (d) T. Hernandezjover, M. Izquierdopulido, M. T. Veciananogues and M. C. Vidalcarou, J. Agric. Food Chem., 1996, 44, 2710; (e) M. Nakamura, T. Sanji and M. Tanaka, Chem.-Eur. J., 2011, 17, 5344; $(f)$ B. Lee, R. Scopelliti and K. Severin, Chem. Commun., 2011, 47, 9639.

6 (a) X. Yang, G. Peter and C. Hauser, Electrophoresis, 2006, 27, 468; (b) X. F. Zhang, X. L. Liu, R. Lu, H. Z. Zhang and P. Gong, J. Mater. Chem., 2012, 22, 1167; (c) W. X. Zhang, Z. X. Chen and Z. H. Yang, Phys. Chem. Chem. Phys., 2009, 11, 6263; (d) X. J. Huang, T. Y. You, T. Li, X. R. Yang and E. K. Wang, Electroanalysis, 1999, 11, 969; (e) L. Feng, C. J. Musto, J. W. Kemling, S. H. Lim and K. S. Suslick, Chem. Commun., 2010, 46, 2037.

7 (a) A. Leino and B. M. Loo, Ann. Clin. Biochem., 2007, 44, 563; (b) W. Putalun, H. Tanaka and Y. Shoyama, Phytochem. Anal., 2005, 16, 370 .

8 (a) Y. Takagai, Y. Nojiri, T. Takase, W. L. Hinze, M. Butsugan and S. Igarashid, Analyst, 2010, 135, 1417; (b) L. J. Fan and W. E. Jones, J. Am. Chem. Soc., 2006, 128, 6784; (c) M. E. Germain and M. J. Knapp, Inorg. Chem., 2008, 47, 9748; (d) D. Ryu, E. Park, D. S. Kim, S. Yan, J. Y. Lee, B. Y. Chang and K. H. Ahn, J. Am. Chem. Soc., 2008, 130, 2394.

9 (a) X. Feng, B. Tong, J. B. Shen, J. B. Shi, T. Y. Han, L. Chen, J. G. Zhi, P. Lu, Y. G. Ma and Y. P. Dong, J. Phys. Chem. B, 2010, 114, 16731; (b) T. Y. Han, X. Feng, B. Tong, J. B. Shi, L. Chen, J. G. Zhi and Y. P. Dong, Chem. Commun., 2012, 48, 416; (c) X. Y. Shi, H. Wang, T. Y. Han, X. Feng, B. Tong, J. B. Shi, J. G. Zhi and Y. P. Dong, J. Mater. Chem., 2012, 22, 19296.

10 (a) J. C. Baum and D. S. McClure, J. Am. Chem. Soc., 1979, 101, 2335; (b) G. M. Florio, E. L. Sibert and T. S. Zwier, Faraday Discuss., 2001, 118, 315; (c) D. E. Poeltl and J. K. McVey, J. Chem. Phys., 1983, 78, 4349; (d) P. B. Bisht, H. Petek, K. Yoshihara and U. Nagashima, J. Chem. Phys., 1995, 103, 5290; (e) S. Maheshwary, U. Lourderaj and N. Sathyamurthy, J. Phys. Chem. A, 2006, 110, 12662; $(f)$ J. Y. Lee, P. C. Painter and M. M. Coleman, Macromolecules, 1988, 21, 954; (g) C. G. Bazuin and F. A. Brandys, Chem. Mater., 1992, 4, 970; (h) T. Kato and J. M. J. Frechet, Macromolecules, 1989, 22, 3818; (i) S. Xiao, Y. Zou, J. Wu, Y. Zhou, T. Yi, F. Lia and C. Huang, J. Mater. Chem., 2007, 17, 2483. 